\title{
Evidências de Validade da Escala de Síndrome Pré-Menstrual
}

\author{
Magno Oliveira Macambira ${ }^{1}$ \\ Universidade Estadual de Feira de Santana, Feira de Santana-BA, Brasil \\ Hudson Golino \\ Department of Psychology, University of Virginia, USA
}

\section{RESUMO}

Os estudos sobre variáveis exclusivamente femininas são raros na literatura nacional. O presente estudo teve como objetivo identificar evidências de validade da Escala de Síndrome Pré-menstrual (ESPm). O instrumento foi construído a partir de descrições de sintomas comumente relatados na literatura especializada e passou pelo crivo de juízes que avaliaram os itens e fatores propostos. Posteriormente, o instrumento foi aplicado em 391 trabalhadoras, por meio de um link disponibilizado virtualmente. Os indicadores de validade foram identificados, a partir da análise fatorial confirmatória, onde a melhor adequação foi apresentada por um modelo bifatorial, com menor qui-quadrado, menos graus de liberdade, além de um RMSEA de 0,04 e um CFI de 0,98. Os dois fatores que compõem a escala final (fator emocional e fator físico) contém ao todo 38 itens.

Palavras-chave: medidas organizacionais e ocupacionais; síndrome pré-menstrual; síndrome disfórica pré-menstrual; mulher; tensão pré-menstrual.

\section{ABSTRACT - Preliminary Pre-Menstrual Syndrome Scale Validation}

Studies on exclusively female variables are rare in national literature. The present study aimed at a preliminary validation of the Premenstrual Syndrome Scale (PmSS). The instrument was elaborated based on proposals from nursing and nutrition specialists and was reviewed by judges who evaluated the proposed items and factors. Subsequently, the instrument was applied to 391 workers through a virtual link. The validity indicators were identified from the confirmatory factor analysis, where the best fit was presented by a two-factor model, with a lower chi-square, fewer degrees of freedom, in addition to a RMSEA of 0.04 and an CFI of 0.98. The two factors that make up the final scale (emotional factor and physical factor) contain a total of 38 items.

Keywords: organizational and occupational measurements; premenstrual syndrome; worker's health; premenstrual dysphoric syndrome.

\section{RESUMEN - Validación Preliminar de la Escala de Síndrome Pre-Menstrual}

Los estudios sobre variables exclusivamente femeninas, no son muy frecuentes en la literatura nacional. El presente estudio, tuvo como objetivo realizar una validación preliminar de la Escala de Síndrome Pre-menstrual (ESPm). El instrumento fue construído a partir de propuestas provenientes de la enfermería y nutrición y pasó por eltamiz de jueces que evaluaron los ítems y factores propuestos. Posteriormente, el instrumento fue aplicado a 391 trabajadoras, a través de um link disponibilizado virtualmente. Los indicadores de validez fueron identificados, a partir de análisis factorial confirmatorio, donde la mejor adecuación fue presentada por un modelo bifactorial, con menor qui-cuadrado, menos grados de libertad, además de un RMSEA de 0,04 y un CFI de 0,98. Los dos factores que componenla escala final (factor emocional y factor físico) contienenen total 38 ítems.

Palabras clave: medidas organizacionales y ocupacionales; síndrome pre-menstrual; salud del trabajador; síndrome disfórico premenstrual.

Os estudos sobre comportamento do trabalhador já se apresentam como parte de uma tradição consolidada de pesquisa e elaboração de estratégias de diagnóstico e intervenção para o contexto organizacional no Brasil e no mundo (Siqueira, 2008). Contanto, apesar do crescente espaço ocupado pela mulher no mercado de trabalho, poucos estudos têm se dedicado a questões exclusivamente femininas.
Ao avaliar os estudos que exploram o universo feminino, nota-se que boa parte do conhecimento produzido vem de estudos que diferenciam a conduta ou a expressão de determinados fenômenos entre os sexos (Magalhães \& Macambira, 2013; Ramos \& Jordão, 2014). Contudo, a mulher representa atualmente uma parcela significativa da população de trabalhadores no Brasil, estando inserida em praticamente todas as funções e assumindo posições 
em toda a estrutura hierárquica nas organizações pública e privada, apesar de ainda cercada por preconceitos e estigmas (Fernandes, 2013).

Neste estudo, elege-se uma variável exclusivamente feminina, a Síndrome Pré-Menstrual (SPM), por se tratar de um fenômeno pouco explorado nos estudos sobre comportamento organizacional, apesar de sugerir um impacto significativo no bem-estar, nas emoções, rendimento e humor das mulheres (Espina, Fuenzalida, \& Urrutia, 2005; Silva, Gigante, Carret, \& Fassa, 2006; Mattia, Santos, \& Bernauer, 2008; Chemin et al., 2012).

A Síndrome Pré-Menstrual (SPM) pode ser descrita como o conjunto de sintomas físicos, emocionais e comportamentais sofridos pela mulher, na semana que antecede o ciclo menstrual e que reduzem de intensidade com o início do fluxo menstrual (Ishii, Nishino, \& Campos, 2009). Epidemiologicamente, 75 a 80\% das mulheres apresentam sintomas durante o referido período, podendo em muitos casos demandar auxílio profissional (Brilhante et al., \& 2010).Vale ressaltar que, apesar de incomum, a literatura descreve episódios de menstruação em mulheres com mais de 51 anos, havendo relatos nacionais da ocorrência de menstruação até os 54 anos de idade (Barcelos, Zanini, \& Santos, 2013).

A SPM, em certas mulheres, pode acarretar em um conjunto de sintomas tão agressivos que, de acordo com o Diagnosticans Statistical Manual of Mental Disorders - quinta edição (APA, 2014), caracteriza-se como uma categoria nosológica específica: o Transtorno Disfórico Pré-menstrual. Esse transtorno é categorizado como um transtorno de humor e apresenta prevalência de 2 a $8 \%$ das mulheres em período fértil, com queixas psíquicas relevantes (Demarque et al., 2013). Essa inserção no principal manual de transtornos psicológicos e psiquiátricos do mundo, caracteriza-se como o reconhecimento clínico de um fenômeno que é comumente identificado no senso comum como um sofrimento menor, vivenciado rotineiramente pela mulher.

$\mathrm{Na}$ SPM, o conjunto de sintomas, de ordem física e psicológica, pode ocorrer com intensidade variada na fase lútea tardia do ciclo menstrual, na semana que precede a menstruação e, geralmente, desaparece alguns dias após o início do fluxo menstrual (Muramatsu, Vieira, Simões, Katayama, \& Nakagawa, 2001). Esse ciclo, é iniciado por estímulos fisiológicos do eixo hipotálamo-hipófise-ovário e controla todo o processo de alterações hormonais. O hipotálamo estimula a produção das gonadotropinas que, por sua vez, determinam a secreção ovariana e a produção dos óvulos. Por fim, após o desenvolvimento do óvulo e consequente ovulação e formação do corpo lúteo, o estrogênio e a progesterona produzidos pela ação das gonadotropinas no tecido ovariano produzem as condições para o processo de descamação do endométrio e a ocorrência do fluxo menstrual (Chemin et al., 2012).
Apesar do aparente caráter fisiológico no controle do ciclo menstrual, outros fatores identificados na literatura também impactam na intensidade dos seus efeitos. Entre eles, a nutrição e características da relação da mulher com o ambiente, mais precisamente, nível de estresse e emoções vivenciadas (Costa, Fagundes, \& Cardoso, 2007).

Em uma revisão de literatura, Souza, Ramos, Hara, Stumpf, e Rocha (2012) identificam 27 estudos sobre o Transtorno Disfórico Pré-Menstrual (TDPM). Os estudos identificados evidenciam uma leve alteração no desempenho cognitivo em mulheres sadias ao longo do ciclo menstrual, obtendo menores pontuações na fase lútea. Entre as variáveis revisadas estão: habilidades visuoespaciais e motoras, atenção e concentração, memória verbal, memória visual, memória de trabalho e tempo de reação. Em comparação com mulheres que apresentavam Síndrome Pré-menstrual e Transtorno Disfórico Pré-menstrual, as sadias apresentavam sintomas levemente mais brandos na fase lútea.

Mas como identificar a intensidade da SPM? Atualmente o recurso mais utilizado são listas de verificações de sintomas, realizadas no exame clínico. Esses checklists apontam a presença ou ausência de sintomas, e não sua intensidade, o que pode gerar equívocos no prognóstico.

Em um estudo que envolveu 4.032 mulheres, em 19 diferentes países, trabalhadoras foram convidadas a participar de um levantamento para identificar o impacto da Síndrome Pré-Menstrual e do Distúrbio Disfórico PréMenstrual no absenteísmo e na percepção de eficiência a partir de escalas de autorrelato. Os resultados apontaram que as mulheres classificadas com Síndrome PréMenstrual e com Síndrome Disfórica Pré-Menstrual, de intensidade moderada à grave alegaram ter um nível de absenteísmo superior, com mais de oito horas de absenteísmo por ciclo menstrual. O resultado do indicador de eficiência no trabalho apresentou a mesma tendência, com índices superiores aos das participantes com SPM leve. (Heinemann, Minh, Lindemann, \& Filonenko, 2012). Apesar de intrigante, o trabalho não apresenta resultados sólidos, tendo em vista que não houve a apresentação de resultados estatísticos robustos para confirmar as conclusões.

A escassez de estratégias para avaliar o nível da SPM e a indicação de que esse fenômeno pode interferir em variáveis importantes como bem-estar, qualidade de vida, capacidade produtiva, entre outros, gerou a necessidade do desenvolvimento de um instrumento válido e confiável para a mensuração da intensidade da síndrome pré-menstrual, sendo útil a diversos objetivos clínicos e de gestão de pessoas, por exemplo. Este estudo tem como objetivo descrever a construção e as evidências de validade da Escala de Sintomas da Síndrome de Tensão Pré-menstrual (ESPm) por meio do uso de análise fatorial confirmatória de diversos modelos estruturais. 
Este estudo faz parte de um projeto maior que envolve a relação dos níveis percebidos de sintomas pré-menstruais em trabalhadoras, com outras variáveis comumente estudadas no campo do comportamento organizacional. No entanto, essa escala não é exclusiva para o contexto organizacional, sendo indicada para a avaliação do fenômeno em qualquer contexto.

\section{Método}

\section{Participantes}

Fizeram parte da pesquisa, mulheres de diferentes estados do Brasil. A amostra foi por obtida por conveniência, respeitando uma busca por variabilidade das participantes, como objetivo de desenvolver uma escala válida que independa da idade e perfil socioeconômico. A coleta em vários estados foi operacionalizada através de coleta virtual por meio da plataforma de pesquisa Survey Monkey.

Participaram ao todo 391 mulheres, com idade entre 17 a 54 anos, sendo que $40,4 \%$ da amostra apresenta uma idade entre 26 a 35 anos, sendo essa classe a mais representativa de toda a amostra, $38,2 \%$ está entre 17 e 25 anos, $18,4 \%$ entre 36 e 45 anos e apenas $2,9 \%$ tinham mais de 45 anos. Em termos de escolaridade, a parcela mais representativa tem ensino superior incompleto $(51,7 \%)$, seguindo de superior completo e ensino médio completo (ambos com 15,2\%), especialistas (14,5\%) e o restante das participantes tem ensino médio incompleto $(3,4 \%)$.

\section{Instrumento}

A Escala de Síndrome Pré-menstrual (ESPm) foi construída a partir da categorização de listas de sintomas característicos dessa fase, na literatura especializada (Muramatsu et al., 2001), em formato de uma escala graduada do tipo Likert. $\mathrm{O}$ instrumento foi aplicado por meio digital, garantindo o anonimato e segurança dos trabalhadores e integridade das informações coletadas. Os dados foram transferidos e analisados no software de estatística computacional R (R Core Team, 2014) com objetivo de identificar evidências iniciais de validade por meio de análise fatorial exploratória e confirmatória.

A proposta inicial da escala continha 41 itens, sendo subdividido em dois fatores, a saber: 1. sintomas físicos, que caracterizam os efeitos físicos causados pela SPM e percebidos pelas trabalhadoras como dor e percepções de alterações corporais no período menstrual; e 2. sintomas emocionais, descritos pelas alterações de humor, emoções, autopercepção de desempenho e controle de comportamentos relacionados ao desempenho no trabalho.

Buscando garantir que todos procedimentos éticos de coleta e análise de dados, todas as participantes assentiram com o Termo de Consentimento Livre e Esclarecido (TCLE) e, quando menor de idade, o termo de assentimento. Todos os procedimentos adotados nesta pesquisa estiveram em conformidade com as orientações éticas previstas.

De forma a avaliar, inicialmente, sua validade de conteúdo, a versão inicial da ESPm foi submetida à análise de juízes (experts na área de síndrome pré-menstrual). Foram utilizados cinco juízes, tendo em vista seu grau de especialidade na área. Todos os itens foram avaliados de acordo com a seguinte escala: +1 (O item refere-se à sintomas de SPM); 0 (Não sei opinar); e -1 (O item não se refere à sintomas de $S P M)$. Posteriormente, os juízes avaliaram a pertinência de cada item em relação ao conjunto de dimensões de qualidade de vida. Assim, foi questionado "A qual fator este item pertence?". Somente foram mantidos os itens que apresentaram no mínimo 80\% de concordância na avaliação dos juízes (Pasquali, 1999). Itens que possuíram grande variação no padrão de avaliação do seu conteúdo foram aperfeiçoados e mantidos na versão operacional da medida, a fim de que os procedimentos estatísticos possam auxiliar na decisão sobre sua validade de construto.

No segundo momento, após a análise de juízes, a escala foi aplicada em sua versão preliminar em algumas trabalhadoras no formato presencial. Esse procedimento, auxiliou na análise semântica e buscou verificar se os itens seriam bem compreendidos pelo público-alvo ou se havia itens que não se adequavam ao nível sociocultural das possíveis participantes da pesquisa. Para a realização da análise semântica, 10 trabalhadoras foram selecionadas intencionalmente, incluindo diferentes níveis educacionais e atividades laborais diversificadas para responder ao instrumento. Foi solicitado que quaisquer dúvidas na formulação dos itens ou do instrumento como um todo fossem expressas abertamente. Os itens enquadrados como extensos, complexos ou de difícil entendimento foram reformulados para a elaboração dos itens de teste da ESPm.

Por fim, a escala elaborada apresenta itens de complemento à afirmação: "No período que antecede a menstruação eu...". Entre os itens temos: "Percebo meu humor alterado", "Tenho pena de mim mesmo", "Tenho dor de cabeça", "Tenho dificuldade de concentração", "Sinto raiva", "Sinto vontade de chorar", entre outros. O questionário foi dividido em três partes. A primeira solicita informações sobre o perfil de trabalho, a segunda apresenta a escala de síndrome pré-menstrual e a teceria parte levanta informações sociodemográficas.

\section{Procedimento de Coleta de Dados}

Os dados foram coletados por meio de uma plataforma virtual de pesquisa: Survey Monkey. Após a elaboração do questionário, foi gerado um link e disponibilizado por e-mail para as participantes locais. As participantes eram convidadas a responder à pesquisa e era dada a liberdade para que elas convidassem novas participantes, respeitando os critérios de inclusão estabelecidos. 

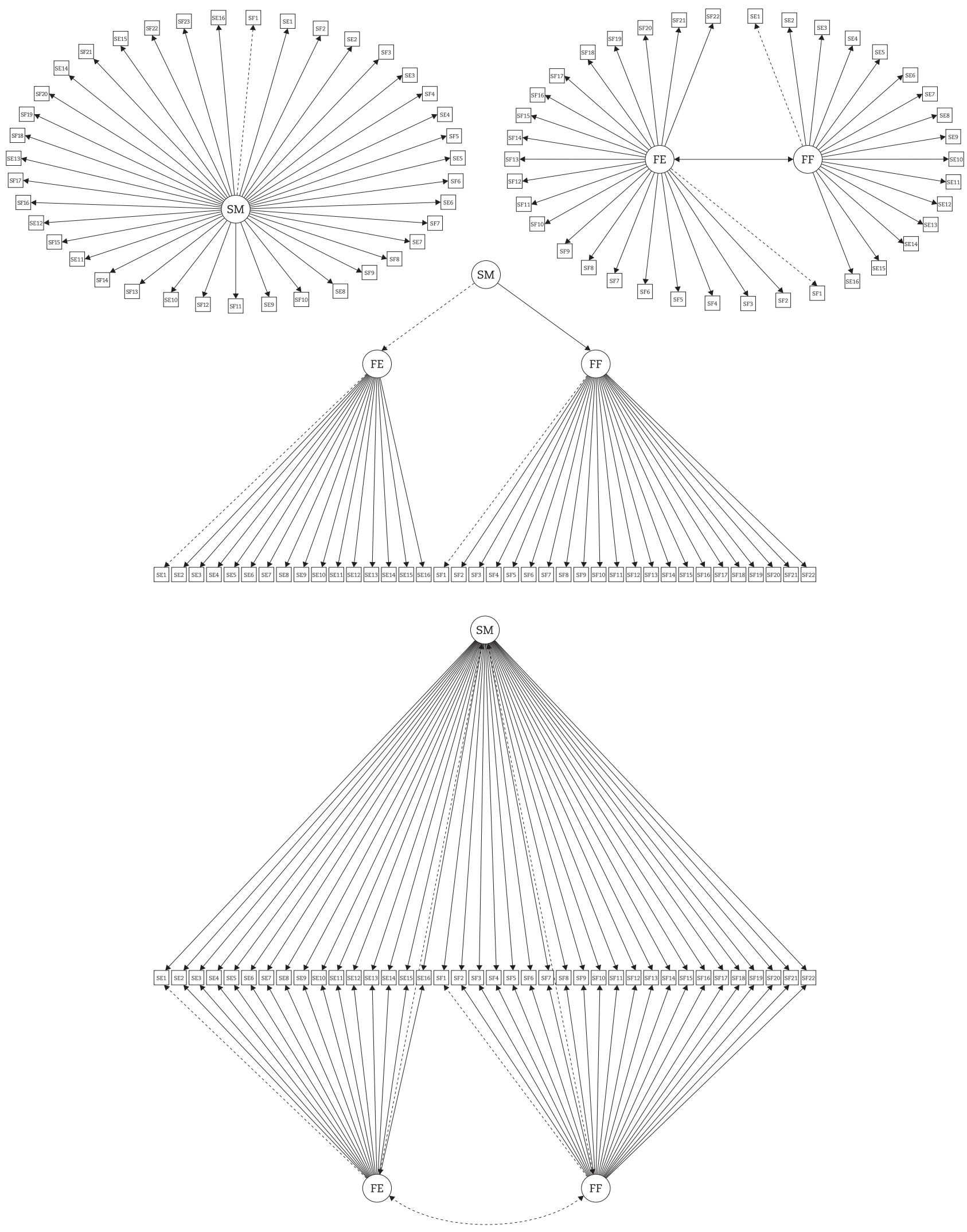

Figura 1. Modelos testados

Legenda. Modelo unidimensional (superior à esquerda), modelo de dois fatores correlacionados (superior à direita), modelo hierárquico (inferior à esquerda) e modelo bifatorial (inferior à direita, a linha pontilhada entre FE e FF aponta uma ausência de correlação, uma vez que os modelos bifatoriais são ortogonalizados) 


\section{Análise de Dados}

A análise fatorial confirmatória foi empregada por meio do pacote lavaan (Rosseel, 2012) do software de estatística computacional R (R Core Team, 2014). Devido à natureza politômica dos dados, utilizou-se o estimador $w l s m v$, que é uma versão robusta do weighted least squares. O ajuste dos dados aos modelos foi verificado por meio dos índices Root Mean Square Error of Approximation (RMSEA) e do Comparative Fit Index (CFI). Um ajuste adequado aos dados é apontado por meio de um RMSEA igual ou menor que 0,05 (Browne \& Cudeck, 1993) e um CFI igual ou maior que 0,95 (Hu \& Bentler, 1999). A Figura 1 apresenta os modelos fatoriais confirmatórios que foram gerados, utilizando-se o pacote SemPlot (Epskamp, 2014). Primeiro verificou-se o ajuste de um modelo unidimensional com todos os itens do instrumento. Em seguida, verificou-se o ajuste de um modelo com dois fatores latentes (FF e
FE), e uma versão hierárquica com duas variáveis latentes de primeiro nível (FF e FE) e uma variável latente de segundo nível (SM). Por último, verificou-se o ajuste de um modelo bifatorial com um fator geral de primeiro nível (SM) explicando todos os itens, e dois fatores específicos (FF e FE), todos ortogonalizados.

\section{Resultados}

A análise fatorial confirmatória mostrou que todos os modelos apresentaram adequado grau de ajuste aos dados. A Tabela 1 apresenta os índices de ajuste dos modelos testados.

No entanto, o modelo bifatorial é o que apresenta o melhor ajuste aos dados, uma vez que possui o menor qui-quadrado, além de um CFI de 0,98 e um RMSEA de 0,04 . O modelo é composto pelos seguintes itens e cargas padronizadas pelo fator específico e o geral (Tabela 2).

Tabela 1

Ajuste dos Modelos Testados por meio de Estimador Não Robusto

\begin{tabular}{lccccc}
\hline \multicolumn{1}{c}{ Modelo } & $\chi^{2}$ & Graus de Liberdade & $p$ & CFI & RMSEA \\
\hline Unidimensional & 1363,41 & 702 & 0,000 & 0,94 & 0,05 \\
Dois fatores & 1229,49 & 664 & 0,000 & 0,95 & 0,05 \\
Hierárquico com dois fatores & 1229,49 & 663 & 0,000 & 0,95 & 0,05 \\
Bifatorial & 842,43 & 627 & 0,000 & 0,98 & 0,04 \\
\hline
\end{tabular}

Tabela 2

Carga Padronizada dos Itens nos Fatores Específicos (Sintomas Físicos e Sintomas Emocionais) e no Fator Geral de Primeiro Nível do Modelo Bifatorial

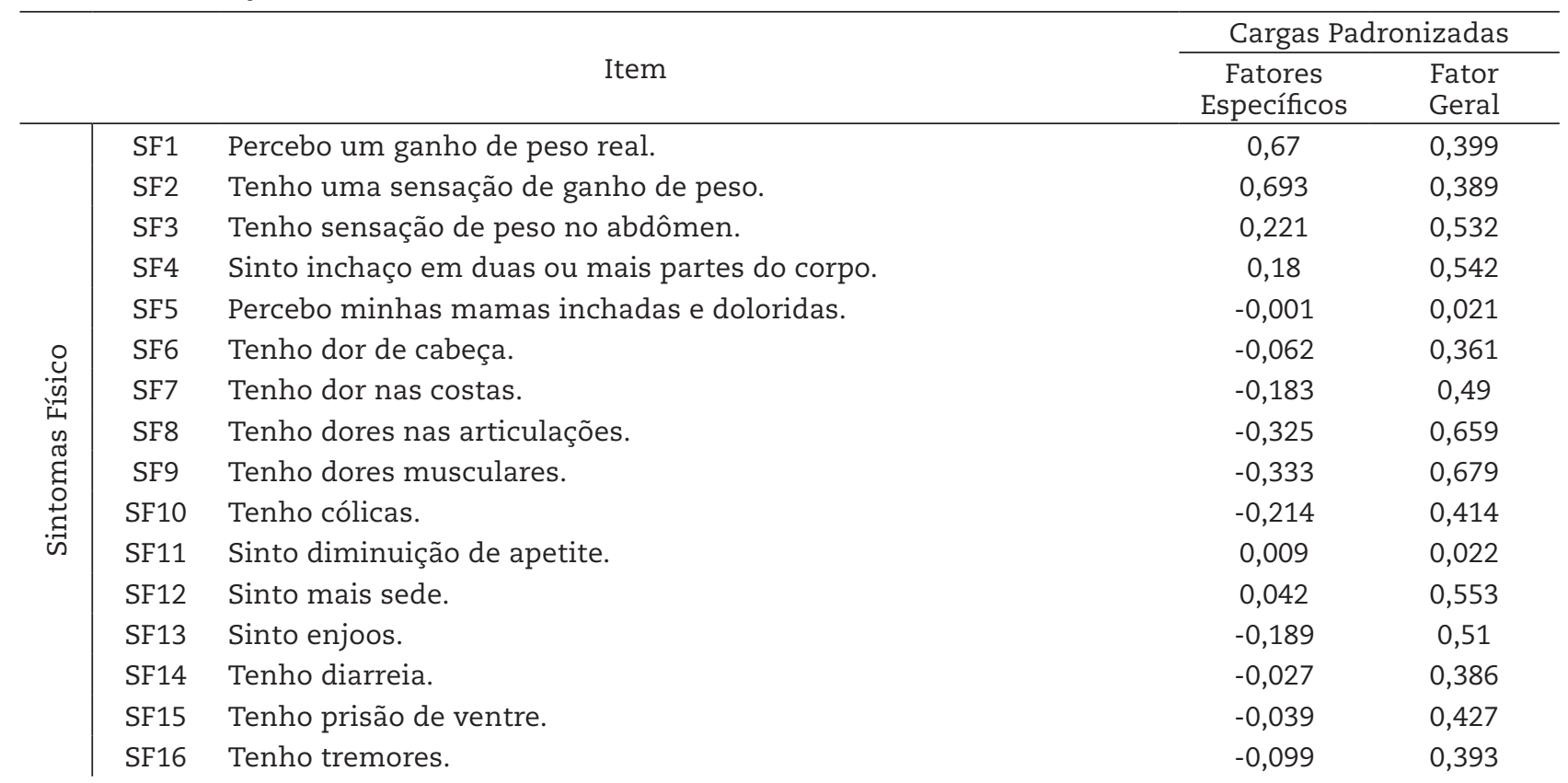


Tabela 2 (continuação)

Carga Padronizada dos Itens nos Fatores Específicos (Sintomas Físicos e Sintomas Emocionais) e no Fator Geral de Primeiro Nível do Modelo Bifatorial

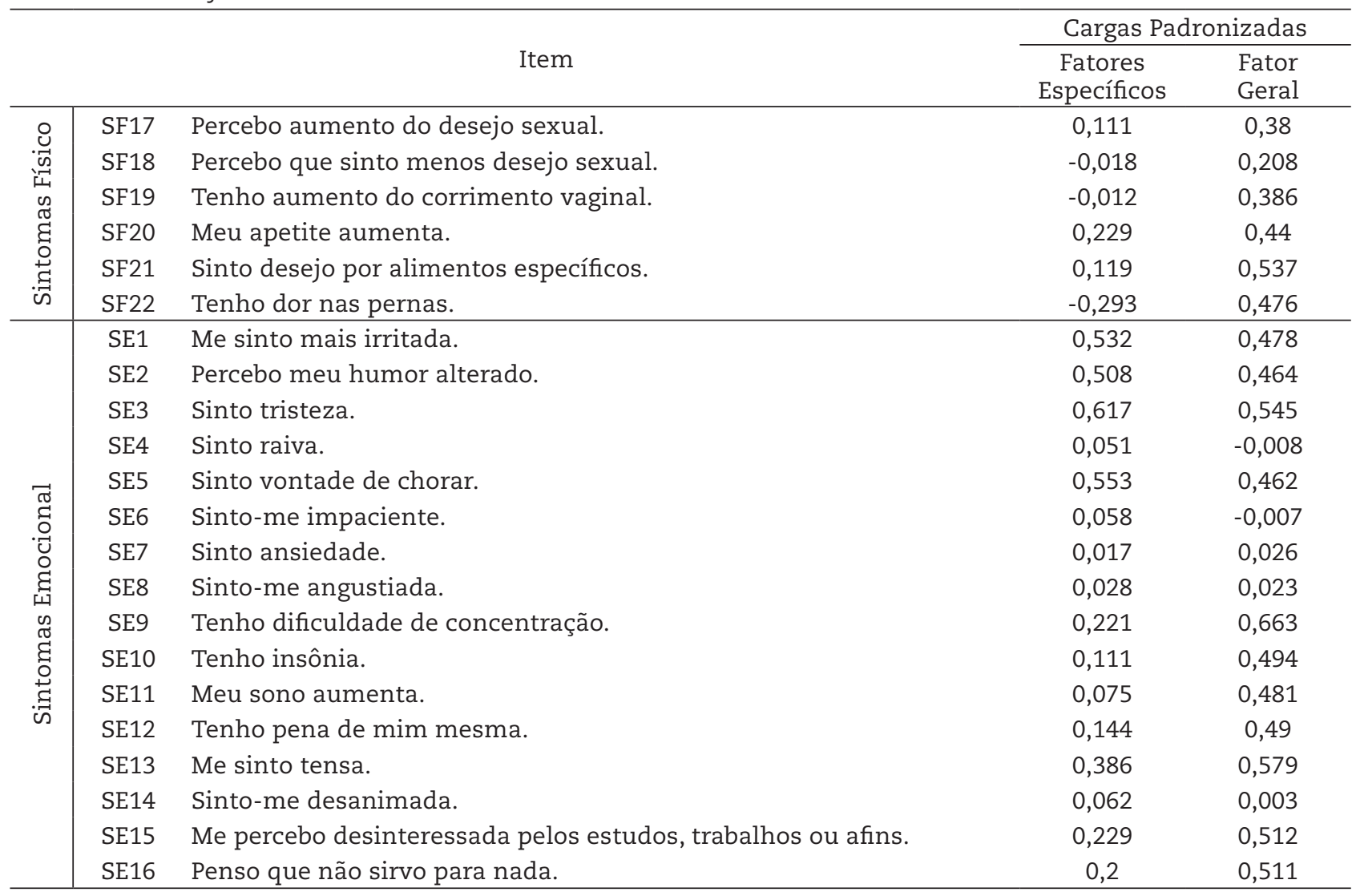

A Figura 2 apresenta as cargas fatoriais padronizadas dos itens nos fatores. Nela é possível visualizar as cargas fatoriais padronizadas para cada um dos dois fatores testados.

Golino e Gomes (2015) apontam uma forma de se verificar a força do fator geral em um modelo bifatorial, por meio do cálculo da variância comum (Explained Common Variance: Reise, Moore, \& Haviland, 2010):

$$
\mathrm{ECV}=\frac{\sum \lambda^{2}{ }_{\mathrm{Gen}}}{\left(\sum \lambda_{\mathrm{Gen}}^{2}\right)+\left(\sum \lambda_{\mathrm{F} 1}^{2}\right)+\left(\sum \lambda_{\mathrm{F} 2}^{2}\right)+\ldots+\left(\sum \lambda_{\mathrm{Fk}}^{2}\right)}
$$

1. onde $\lambda^{2}$ Gen é o quadrado das cargas não padronizadas do fator geral e $\lambda_{F k}^{2}$ é o quadrado das cargas não padronizadas para cada uma das k variáveis latentes. A ECV do modelo bifatorial mostra que o fator geral de primeiro nível explica por volta de $51 \%$ da variância comum dos itens. O restante, $49 \%$ é explicado pelos fatores específicos (fator físico e fator emocional).

\section{Discussão}

A análise confirmatória da escala de Síndrome PréMenstrual demonstrou ser promissora no esforço em identificar a intensidade desse fenômeno em trabalhadoras. A escala final é composta por 38 itens, divididos em dois fatores específicos (físicos e emocionais), além de um fator geral de primeiro nível, com bons indicadores na análise fatorial confirmatória. É interessante que o modelo com melhor ajuste tenha sido o modelo bifatorial, uma vez que evidencia que é preciso considerar, não apenas as especificidades dos sintomas físicos e emocionais, mas também a especificidade de um fator único, geral, de condições da síndrome pré-menstrual.

De acordo com Golino e Gomes (2015), há um conjunto de vantagens de se utilizar o modelo bifatorial na busca pela melhor estrutura de instrumentos de avaliação. Dentre essas vantagens, vale elencar que esse modelo permite que tanto fatores específicos quando um fator geral de primeiro nível, compitam pela explicação 
da variância dos dados, sendo uma estratégia adequada para investigar se os itens de um instrumento avaliam um fator único comum. O resultado evidenciou que o fator geral explica $51 \%$ da variância comum dos itens, não sendo um fator suficientemente robusto para considerar o instrumento unidimensional. Os fatores específicos e o fator geral dividem quase que de forma igual a explicação da variância dos itens do instrumento.

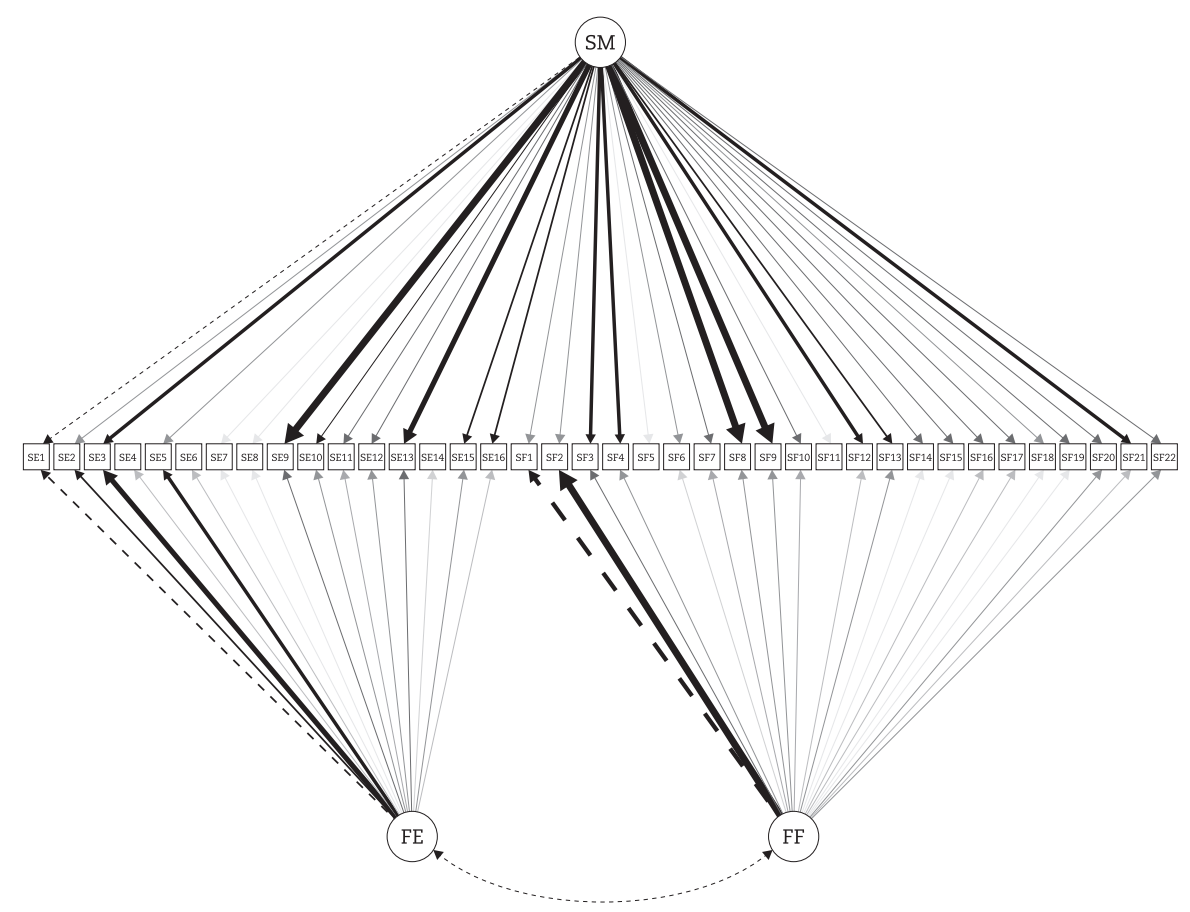

Figura 2. Cargas fatoriais padronizadas para dois fatores

Legenda. Os valores relacionados à Figura 2 estão na Tabela 2

Os dois fatores identificados pela análise confirmatória refletem dois grandes conjuntos de sintomas da SPM, a saber: 1. percepção de sintomas físicos - relacionados à retenção de líquido, alteração no apetite, dores pelo corpo, cefaleia e mastodinia, comumente conhecida com dor nas mamas, entre outras alterações no corpo ou funcionalidade do organismo; 2 . percepção de sintomas emocionais - que podem ser compreendidos como a vivência de estados emocionais alterados.

A literatura que aborda a discussão sobre os sintomas da síndrome pré-menstrual e os dois fatores evidenciados na escala elaborada, ainda, é muito pautada na percepção que as mulheres têm no período em que ela se apresenta. Esses sintomas remetem à origem orgânica, assim como a interação entre o sujeito e seu contexto, que se forma em função de histórico de aprendizagem individual e social, nível de resiliência frente aos efeitos da SPM e estratégias de enfrentamento.

Este estudo abre um campo de possibilidades para investigação dos efeitos e relações entre a síndrome pré-menstrual e outras variáveis de comportamento social, incluindo interações com colegas, estereótipos, entre outras variáveis já citadas anteriormente que podem moderar a percepção dos sintomas eliciados pela SPM.

Outra agenda possível, diz respeito aos preditores dos sintomas emocionais. Respeitando a base hormonal e suas influências físicas, que fatores psicossociais poderiam moderar o aumento ou redução desses sintomas? As questões apontadas nos direcionam para uma agenda de pesquisas de antecedentes, possíveis correlatos e consequentes em diferentes níveis de análise (individuais e grupais) e contextos (escola, família, organizações, entre outros) que podem se relacionar com a intensidade percebida dos sintomas pré-menstruais.

A forma de coleta (on-line), pode ser identificada com uma limitação do trabalho, pois, apesar da conveniência para as participantes e segurança quando a confidencialidade das informações comuns às estratégias de coletas on-line (Vieira, Castro, \& Schuch Junior, 2010), essa estratégia permite uma gama de equívocos no processo de preenchimento da escala, ausência de padronização sobre o contexto de aplicação, abandono do questionário durante o preenchimento, entre outros eventos que podem resultar em dados não confiáveis. Essa mesma limitação, sugere que, em futuras pesquisas, deva-se testar a estabilidade da escala em coletas presenciais. 


\section{Referências}

American Psychiatric Association (2014). Diagnostic and Statistical Manual of Mental Disorders. 5th. ed, Porto Alegre: Artmed.

Barcelos, R. S., Zanini, R. V., \& Santos, I. S. (2013). Distúrbios menstruais entre mulheres de 15-54 anos de idade em Pelotas, Rio Grande do Sul, Brasil: estudo de base populacional. Caderno de Saúde Pública, 29(11), 2333-2346. doi: 10.1590/0102-311x00002813

Brilhante, A. V. M., Bilhar, A. P. M., Carvalho, C. B., Karbage, S. A. L., Pequeno Filho, E. P., \& Rocha, E. S. (2010). Síndrome prémenstrual e síndrome disfórica pré-menstrual: aspectos atuais. Feminina, 38(7), 373-378. Recuperado de http://files.bvs.br/ upload/S/0100-7254/2010/v38n7/a373-378.pdf

Browne, M. W., \& Cudeck, R. (1993). Alternative ways of assessing model t. Em K. A. Bollen. \& J. S. Long (Eds.), Testing structural equation models (pp. 136-162). Newbury Park, CA: Sage.

Chemin, S. M., Silva, S., Fernanda, B., Silva, C., Farina, B.V., Spinoza, E. D., \& Breda, S. M. (2012). Influência da tensão pré-menstrual no consumo alimentar. Revista Nutrire: Sociedade Brasileira de Alimentação e Nutrição, 37(1), 13-21. doi: 10.4322/nutrire.2012.002

Costa, Y. R., Fagundes, R. L. M., \& Cardoso, B. R. (2007). Ciclo menstrual e consumo de alimentos. Revista Brasileira Nutrição Clínica, 22(3), 203-209.

Demarque, R., Rennó Junior, J., Ribeiro, H. L., Cavalsan, J. P., Rocha, R., Cantilino, A., Ribeiro, J. A. M., Valadares, G., \& Silva, A.G. (2013). Transtorno disfórico pré-mentrual: um breve panorama. Revista de Debates em Psiquiatria, 3(5), 6-11. Recuperado de https://docplayer. com.br/18971511-Artigos-transtorno-disforico-pre-menstrual-um-breve-panorama.html

Epskamp, S. (2014). SemPlot: Path diagrams and visual analysis of various SEM packages' output. Recuperado dehttp://CRAN.R-project.org/ package $=$ semPlot

Espina, N., Fuenzalida, A., \& Urrutia, M. T. (2005). Relación entre rendimiento laboral y síndrome premenstrual. Revista Chilena de Obstetricia y Ginecología. 70(2), 113-118.doi: 10.4067/S0717-75262005000200011

Fernandes, M. A. (2013). A inserção da mulher no mercado de trabalho: um estudo sob a perspectiva da psicologia. Gestão E Conhecimento, $s / v(1), 1-20$. Recuperado de https://www.pucpcaldas.br/graduacao/administracao/revista/artigos/v2013/Artigo01 2013.pdf

Golino, H, F., \& Gomes, C.M. (2015). Investigando estágios de desenvolvimento do raciocínio indutivo usando a análise fatorial confirmatória, o Modelo Logístico Simples de Rasch e o modelo de teste logístico linear (Rasch Estendido). Em H. F. Golino, C. M. Gomes, A. Amantes, \& G. Coelho, Psicometria Contemporânea: Compreendendo os Modelos Rasch (pp.283-331). São Paulo: Casa do Psicólogo/Pearson.

Hu, L. T., \& Bentler, P. (1999). Cutoff criteria for fit indexes in covariance structure. Structural Equation, 6(1), 1-55.doi: 10.1080/10705519909540118

Heinemann, L. A. J, Minh, T., Heinemann, K., Lindemann, M., \& Filonenko, A. (2012). Intercountry Assessment of the Impact of Severe Premenstrual Disorders on Work and Daily Activities. Health Care for Women International, 33(2), 109-124.doi: 10.1080/07399332.2011.610530

Ishii, C., Nishino, L. K., \& Campos, A. H. (2009). Caracterização vestibular no ciclo menstrual. Brazilian Journal of Otorhinolaryngology, 75(3), 375-80. doi: 10.1016/S1808-8694(15)30655-8

Magalhães, M. O., \& Macambira M. O. (2013). Estilos interpessoais e vínculo com a organização: diferenças de gênero. Psico, 44(1), 92-102. Recuperado de http://revistaseletronicas.pucrs.br/ojs/index.php/revistapsico/article/view/10660/8852

Mattia, A. L., Santos, C. M., Telles, B., \& Bernauer, M. C. (2008). Síndrome pré-menstrual: Influências na equipe de enfermagem de centro cirúrgico. O Mundo da Saúde. 32(4), 495-505. Recuperado de https:/www.saocamilo-sp.br/pdf/mundo_saude/65/11_Sindrome_baixa.pdf

Muramatsu C. H., Vieira, O. C. S., Simões, C. C., Katayama, D. A., \& Nakagawa, F. H. (2001). Consequềncias da síndrome de tensão prémentrual na vida da mulher. Revista da Escola de Enfermagem da USP, 35(3), 205-13. doi: 10.1590/S0080-62342001000300002

Pasquali, L. (1999). Taxonomia dos instrumentos psicológicos. Em Pasquali, L. (Ed.). Instrumentos Psicológicos: manual prático de elaboração (pp.27-36) Brasília: LabPAM-IBAPP.

R Core Team. (2014). R: A language and environment for statistical computing. R Foundation for Statistical.

Ramos, V., \& Jordão, F. (2014). Género y estrés laboral: Semejanzas y diferencias de acuerdo a factores de riesgo e mecanismos de coping. Revista Psicologia: Organizações e Trabalho, 14(2), 218-229. Recuperado de http://pepsic.bvsalud.org/scielo.php?script=sci_arttext\&pid $=\mathrm{S} 1984-66572014000200008$

Reise, S. P., Moore, T. M., \& Haviland, M. G. (2010). Bifactor models and rotations: Exploring the extent to which multidimensional data yield univocal scale scores. Journal of Personality Assessment, 92(6), 544-559.doi: 10.1080/00223891.2010.496477

Rosseel, Y. (2012). lavaan: An R Package for Structural Equation Modeling. Journal of Statistical Software, 48(2), 1-36. Recuperado de http:// www.jstatsoft.org/v48/i02/

Siqueira, M. M. M. (2008). Satisfação no trabalho. Em M. M. M. Siqueira (Ed.), Medidas do comportamento organizacional: ferramentas de diagnóstico e gestão (pp. 265-274). Porto Alegre: Artmed.

Silva C. M. L., Gigante D. P., Carret M. L. V., \& Fassa A. G. (2006). Population study of premenstrual syndrome. Revista Saúde Pública, 40(1), 47-56. doi: 10.1590/S0034-89102006000100009

Souza, E. G., Ramos, V. M. G., Hara, C., Stumpf, B. P., \& Rocha, F. L. (2012). Neuropsychological performance and menstrual cycle: A literature review. Trends Psychiatry Psychother, 34(1), 5-12.doi: 10.1590/S2237-60892012000100003

Vieira, H. C., Castro, A. E., \& Schuch Junior, V. F. (2010). O uso de questionário via e-mail em pesquisas acadêmicas sob a ótica dos respondentes. XIII Seminários de Administração-SemeAd. São Paulo. Recuperado de https://bit.ly/2y5DnCn

\section{Sobre os autores}

Magno Oliveira Macambira é doutor em Psicologia pela Universidade Federal da Bahia e professor auxiliar da Universidade Estadual de Feira de Santana.

Hudson Golino é doutor em Neurociências pela Universidade Federal de Minas Gerais e professor assistente do Programa de Métodos Quantitativos do Departamento de Psicologia da Universidade da Virgínia (EUA). 